\title{
Otimização da prensagem a frio de grãos de amendoim em prensa contínua tipo expeller
}

\author{
Optimization peanut grain expelling
}

\author{
Anna Leticia Montenegro Turtelli PIGHINELLI ${ }^{1 \star}$, Kil Jin PARK ${ }^{1}$, Ana Maria RAUEN ${ }^{2}$, \\ Gabriela BEVILAQUA ${ }^{1}$, João André Guillaumon FILHO ${ }^{1}$
}

\section{Resumo}

A prensagem contínua de grãos oleaginosos é um método rápido, fácil e de baixo custo para a obtenção de óleos, sendo uma alternativa viável para pequenas comunidades de agricultores. A eficiência deste método é bem inferior àquela conseguida pelo tradicional método de extração por solvente, sendo afetada diretamente pelas condições iniciais dos grãos, como o teor de umidade e temperatura, e por aspectos construtivos da prensa, como dimensionamento do seu eixo e da gaiola. No presente trabalho, avaliou-se por meio de um planejamento experimental, aliado à metodologia de superfície de resposta, a influência da temperatura e do teor de umidade dos grãos de amendoim no rendimento em óleo bruto. As faixas avaliadas foram entre 8 e $10 \%$ para o teor de umidade e entre 25 e $60{ }^{\circ} \mathrm{C}$ para a temperatura. Os óleos brutos obtidos foram caracterizados conforme metodologia oficial da AOCS. As duas variáveis estudadas foram significativas para o nível de confiança adotado (95\%), sendo as condições ótimas localizadas próximas aos maiores valores de temperatura e teor de umidade dos grãos. A faixa de temperatura de 50 a $65^{\circ} \mathrm{C}$ e o teor de umidade de 10 a $10,8 \%$ correspondem a rendimentos máximos.

Palavras-chave: Arachis hypogaea L.; superfície de resposta; rendimento em óleo bruto; caracterização química.

\begin{abstract}
The continuous oilseed expeller is a quick, easy, and low cost method to obtain oils, so it is used in small agriculture communities. This method is less efficient than the traditional solvent extraction, since it is affected directly by the initial conditions of the raw material, such as grain moisture temperature, and also by some machine features, such as the shaft and barrel design. In this work, the influence of the temperature and moisture content of peanut grains on the yield of gross oil was evaluated by experimental design and response surface methodology. The studied temperature range was between 8 and 10\%, and the moisture content range was between 25 and $60{ }^{\circ} \mathrm{C}$. The unrefined oil obtained was analyzed by the AOCS official method. The two studied variables were significant at $95 \%$ level, and the optimal condition values were close to the highest values of temperature and moisture content of grains. The temperature range from 50 to $65^{\circ} \mathrm{C}$ and the moisture content from 10 to $10.8 \%$ correspond to the maximum oil yields.
\end{abstract}

Keywords: Arachis hypogaea L.; response surface; unrefined oil yield; chemical characterization.

\section{Introdução}

O programa nacional de biodiesel, com a obrigatoriedade da mistura de $2 \%$ ao diesel de petróleo, gerou um aumento na demanda por óleos vegetais, uma das principais matérias-primas para a produção deste combustível renovável. As pequenas comunidades rurais são atualmente fornecedoras de grãos oleaginosos, constituindo apenas uma pequena parte do programa governamental. Para aumentar a participação dessas comunidades no programa uma opção seria possibilitar aos agricultores a extração de óleos vegetais em suas propriedades, usando prensas mecânicas contínuas (PIGHINELLI, 2007).

São três os métodos de extração de óleo, podendo sofrer algumas modificações ou mesmo serem utilizados combinados entre si: prensa hidráulica por batelada; prensa mecânica contínua (expeller); e extração por solventes. As prensas hidráulicas estão sendo substituídas por prensas mecânicas, mais eficientes na extração do óleo, com funcionamento simples e baixo custo de aquisição e manutenção, sendo recomendadas para pequenas cooperativas (WEISS, 1983).
Os expellers possuem capacidade de processamento variando entre 40 e $1000 \mathrm{~kg} / \mathrm{h}$. As prensas de pequena capacidade, como as de $40 \mathrm{~kg} / \mathrm{h}$, embora sejam as mais indicadas para as pequenas propriedades rurais, apresentam uma baixa eficiência, deixando entre 8 e 14\% de óleo na torta (SRIKANTHA, 1980 apud SINGH; BARGALE, 2000).

O rendimento em óleo é afetado por parâmetros construtivos da prensa, como dimensionamentos do eixo sem fim e da gaiola, pressão aplicada sobre a massa de grãos e também pelo preparo prévio da matéria-prima a ser processada, como temperatura de prensagem e teor de umidade das amostras (WIESENBORN et al., 2001). Para melhorar a eficiência das prensas de pequena capacidade, estão sendo feitos estudos de otimização das principais variáveis envolvidas no processo (OHLSON, 1992 apud SINGH; BARGALE, 2000).

Singh et al. (2002), em seus estudos de otimização, relataram a importância de se definir uma faixa ótima para o teor de

${ }^{1}$ Faculdade de Engenharia Agrícola - FEAGRI, Universidade Estadual de Campinas - UNICAMP, Cidade Universitária Zeferino Vaz, CP 6011, CEP 13083-875,

Campinas - SP, Brasil,E-mail: annalets@agr.unicamp.br; kil@agr.unicamp.br; gabriela.bevilaqua@gmail.com; joaoagf@terra.com.br

2 Instituto de Tecnologia de Alimentos - ITAL, Av. Brasil, 2880, CP 139, CEP 13070-178, Campinas - SP, Brasil, E-mail: anarauen@ital.sp.gov.br

${ }^{*}$ A quem a correspondência deve ser enviada 
umidade, já que foi observado que valores muito altos reduzem a fricção da massa de grãos, causando um baixo rendimento, já valores muito baixos prejudicam o funcionamento da prensa. Foi concluído que uma diminuição no teor de umidade e um aumento na temperatura melhoram o rendimento em óleo.

O planejamento experimental é uma ferramenta utilizada para minimizar o número de experimentos e otimizar os processos, tendo como base a análise estatística. Um bom planejamento consiste em projetar um experimento de forma que seja capaz de fornecer exatamente o tipo de informação que se procura e, para tanto, deve-se conhecer muito bem o processo em que se deseja trabalhar, e analisar os fatores e as respostas de interesse para o mesmo. Os fatores são as variáveis que podem ser controladas no processo e as respostas são as variáveis de saída do sistema nas quais se tem interesse, que podem ou não ser afetadas por modificações provocadas nos fatores. Quando ainda se tem o interesse de otimizar um processamento, isto é, maximizar ou minimizar algum tipo de resposta, utiliza-se a metodologia da superfície de resposta (BARROS NETO; SCARMINIO; BRUNS, 2003).

A metodologia da superfície de resposta é uma coleção de técnicas estatísticas e matemáticas usadas para desenvolver, melhorar e otimizar processos, dimensionar e formular produtos, amplamente utilizada no mundo industrial, em situações nas quais se têm diversas variáveis de entrada (chamadas de variáveis independentes) que influenciam uma resposta, como por exemplo, o rendimento do produto ou processo (MYERS; MONTGOMERY, 2002).

De acordo com Barros Neto, Scarminio e Bruns (2003), a metodologia da superfície de resposta é composta de duas etapas: modelagem e deslocamento. A primeira ajusta modelos lineares ou quadráticos a resultados experimentais obtidos de planejamentos experimentais. A segunda busca o caminho de máxima inclinação de um determinado modelo, que é o caminho onde a resposta varia de forma mais pronunciada.

Se $n$ variáveis controladas estão envolvidas, o planejamento necessita $2 n$ ensaios diferentes. Calculam-se os efeitos principais e de interação das variáveis independentes sobre as respostas, determinando aqueles mais significativos e comparando-os com o valor do efeito e o erro experimental estimado. Quando o modelo de primeira ordem não for eficiente, realizam-se ensaios nos pontos axiais para um modelo de segunda ordem (planejamento experimental do tipo central composto).

A obtenção dos modelos empíricos por regressões lineares e não lineares segue uma análise de variância, utilizando como parâmetros: coeficiente de determinação $\left(\mathrm{R}^{2}\right)$ e valor estimado do teste $F$ (BOX; HUNTER; HUNTER, 1978). A análise dos resíduos também é importante para avaliar a qualidade do ajuste. Valores residuais altos indicam má qualidade no ajuste do modelo (BARROS NETO; SCARMINIO; BRUNS, 2003).

Pesquisas agronômicas mostraram que a rotação da cultura da cana-de-açúcar com o amendoim (Arachis hypogaea L.) é uma prática benéfica e que reduz os custos de produção agrícola. Com isso, o estado de São Paulo, grande produtor de cana, voltou a ser um grande produtor de amendoim, respondendo por quase 90\% do volume colhido no Brasil (FREITAS et al., 2005).
A cultivar Runner IAC 886 possui crescimento rasteiro e ciclo de 130 dias nas condições do estado de São Paulo. Tem alta produtividade, exigindo solos com boa fertilidade e controle de doenças, sendo recomendada para cultivo que envolva sistema de produção com alto nível tecnológico. Suas vagens contêm dois grãos, com rendimentos entre 70 e $80 \%$ no descascamento e massa variando entre 0,5 e 0,7 g/grão. Os grãos possuem película de cor rosada e alto teor de óleo (próximo a 50\%). (GODOY et al., 2003 apud SANTOS; GODOY; FÁVERO, 2005).

O presente trabalho visou otimizar o processo de prensagem mecânica contínua a frio em miniprensa modelo MPE - 40 Ecirtec, sendo avaliado o efeito das variáveis independentes de temperatura e o teor de umidade de grãos de amendoim no rendimento de extração de óleo bruto, além de sua caracterização química.

\section{Material e métodos}

\subsection{Matéria-prima}

Os grãos de amendoim, variedade Runner IAC 886, foram fornecidos pela Copercana. Para cada ensaio de prensagem foram utilizados 9,3 kg de grãos (massa seca). O teor de umidade inicial dos grãos de amendoim era de 7,3\% (bs), sendo necessária uma adequação da matéria-prima aos níveis de umidade contidos no planejamento experimental. Para isso, as amostras foram separadas e umidificadas com a quantidade necessária de água para atingirem os teores de umidade de estudo e então foram acondicionadas em sacos plásticos lacrados. As amostras foram deixadas em câmara fria a $10^{\circ} \mathrm{C}$ por um período de 10 dias, até atingirem o equilíbrio.

\subsection{Planejamento experimental}

No planejamento experimental foram utilizadas duas variáveis independentes: o teor de umidade e a temperatura dos grãos, e como variável dependente (resposta), o rendimento em óleo bruto. Os níveis de cada variável foram determinados por um teste preliminar, encontrando assim a faixa mais adequada para a prensa operar normalmente, ou seja, para que a massa de grãos consiga se deslocar adequadamente ao longo do eixo helicoidal.

O planejamento experimental empregado foi o de dois níveis para cada variável independente (teor de umidade e temperatura dos grãos), $2^{2}=4$ experimentos, e com triplicata do ponto central para a avaliação do erro puro (Tabela 1).

\subsection{Extração do óleo}

A extração do óleo bruto foi realizada por prensagem a frio em prensa do tipo expeller, modelo MPE - 40, marca ECIRTEC, com processamento de 40 a $60 \mathrm{~kg} \cdot \mathrm{h}^{-1}$ de matéria-prima.

Os grãos foram retirados da câmara fria, deixados à temperatura ambiente e posteriormente aquecidos até às temperaturas referenciadas no planejamento experimental. Esse aquecimento foi feito em aquecedor de resistências elétricas e com sistema de agitação. 
Terminado o aquecimento, os grãos foram colocados no expeller, sendo iniciada a prensagem, com cada batelada durando em média 10 minutos. $\mathrm{O}$ óleo bruto e a torta foram pesados, sendo o óleo posteriormente filtrado em papel de filtro e caracterizado, enquanto a torta foi avaliada quanto ao seu teor de lipídios.

O cálculo do rendimento em óleo bruto foi feito pela razão entre a massa de óleo obtida na prensagem e a massa de óleo inicial existente no grão. $\mathrm{O}$ rendimento em torta foi feito pela razão entre a massa de torta obtida na prensagem e a massa inicial de grãos.

\subsection{Análises físico-químicas}

O teor de umidade dos grãos foi determinado pelo método da estufa (marca FANEM modelo 320 - SE), por 24 horas, à temperatura de $105^{\circ} \mathrm{C}$, de acordo com a Association of Official Analytical Chemists - AOAC (1998).

O teor de lipídios foi determinado nos grãos e na torta resultante da prensagem, extraindo-se sua matéria graxa com éter de petróleo em um extrator tipo BUTT (extração direta). O método utilizado foi o Bc 3-49, descrito na American Oil Chemists Society - AOCS (1998).

A determinação da cor das amostras de óleo foi feita por comparação com padrões de cores, seguindo a metodologia oficial Cc 13b-45 da AOCS (1998), sendo as leituras feitas no tintômetro Lovibond.

O índice de refração de um óleo está relacionado ao grau de saturação das ligações, com seu teor de ácidos graxos livres, oxidação e tratamento térmico da amostra. Seguiu-se o método oficial Cc 7-25 da AOCS (1998). As leituras foram feitas em refratômetro resfriado com banho (marca Marconi) para se manter a temperatura de $20^{\circ} \mathrm{C}$.

O método utilizado na determinação do teor de ácidos graxos livres existentes na amostra, por titulometria, é o Ca 5a-40 da AOCS (1998).

O índice de iodo foi determinado pelo Método de Wijs, conforme o método Cd 1-25 da AOCS (1998). Este índice mede a insaturação de óleo e gorduras e é expresso em termos do número de centigramas de iodo absorvidos por grama de amostra (percentagem de iodo absorvido).

Tabela 1. Planejamento experimental empregado no estudo de prensagem de grãos de amendoim.

\begin{tabular}{lcccccc}
\hline \multirow{2}{*}{ Ensaios } & \multicolumn{2}{c}{ Variável codificada } & & \multicolumn{2}{c}{ Variável real } \\
\cline { 3 - 4 } \cline { 6 - 7 } & & $\mathrm{X}_{1}$ & $\mathrm{X}_{2}$ & & $\mathrm{~T}\left({ }^{\circ} \mathrm{C}\right)$ & $\mathrm{U}(\%)$ \\
\hline Pontos & 1 & -1 & -1 & & 25,0 & 8,0 \\
fatoriain & 2 & +1 & -1 & & 60,0 & 8,0 \\
& 3 & -1 & +1 & & 25,0 & 10,0 \\
& 4 & +1 & +1 & & 60,0 & 10,0 \\
Pontos & 5 & 0 & 0 & & 42,5 & 9,0 \\
centrais & 6 & 0 & 0 & & 42,5 & 9,0 \\
& 7 & 0 & 0 & & 42,5 & 9,0 \\
\hline
\end{tabular}

$\mathrm{X}_{1}$ : valores codificados da temperatura; $\mathrm{X}_{2}$ : valores codificados do teor de umidade;

$\mathrm{T}$ : valores reais de temperatura; e $\mathrm{U}$ : valores reais do teor de umidade.
O índice de saponificação é a quantidade de álcali necessária para saponificar uma quantidade definida de amostra. É expresso como o número de miligramas de hidróxido de potássio $(\mathrm{KOH})$ necessário para saponificar $1 \mathrm{~g}$ de amostra. A determinação foi feita conforme método oficial Cd 3-25 da AOCS (1998).

O índice de peróxido determina todas as substâncias que oxidam o iodeto de potássio (KI), em miliequivalentes de peróxido por $100 \mathrm{~g}$ de amostra, sob as condições de teste. Essas substâncias são geralmente consideradas como peróxidos ou outros produtos similares da oxidação de gorduras. O método para esta análise é o Cd 8-53 da AOCS (1998).

A densidade relativa de um óleo determina a relação da massa de um volume unitário da amostra a $20^{\circ} \mathrm{C}$ e da massa de um volume unitário da água a $20^{\circ} \mathrm{C}$, conforme mostrado no método oficial Cc 10a-25 da AOCS (1998).

As matérias insaponificáveis incluem aquelas substâncias freqüentemente encontradas dissolvidas nos óleos e gorduras, as quais não se saponificam com os álcalis cáusticos, mas são solúveis em solventes comuns de gorduras. Estão incluídos álcoois alifáticos, esteróis, pigmentos e hidrocarbonetos. O método oficial é Ca 6a-40 da AOCS (1998).

A composição em ácidos graxos feita em cromatógrafo gasoso, conforme métodos Ce 1e-91; Ce 1F-96; Ce 1-62 e Ce 2-66 da AOCS (1998) e método 996.06 da AOAC (2000).

\subsection{Modelo matemático}

A resposta, rendimento em óleo bruto, foi expressa por um modelo contendo termos de primeira ordem, conforme a equação não codificada, Equação 1.

$\operatorname{Re} n d=b_{o}+b_{1} * T+b_{2} * U+b_{12} * T * U$

A variável de resposta medida é Rend, que foi analisada por múltipla regressão através do método que utiliza erro padrão para ajustar a Equação 1, no software estatístico Statistica versão 6.0 (2001).

\section{Resultados e discussão}

O teor de lipídios nos grãos de amendoim foi de $44,78 \%$. Na Tabela 2 estão mostrados os resultados obtidos com a extração de óleo bruto em expeller.

A Tabela 3 apresenta os efeitos lineares dos parâmetros, bem como suas interações na avaliação do rendimento em

Tabela 2. Rendimento em óleo bruto de amendoim para cada condição experimental.

\begin{tabular}{llccc}
\hline \multicolumn{1}{c}{ Ensaios } & $\mathrm{T}\left({ }^{\circ} \mathrm{C}\right)$ & $\mathrm{U}(\%)$ & Óleo bruto (\%) \\
\hline Pontos & 1 & 25,0 & 8,0 & 4,80 \\
fatoriais & 2 & 60,0 & 8,0 & 40,82 \\
& 3 & 25,0 & 10,0 & 24,01 \\
& 4 & 60,0 & 10,0 & 74,44 \\
Pontos & 5 & 42,5 & 9,0 & 28,81 \\
centrais & 6 & 42,5 & 9,0 & 36,02 \\
& 7 & 42,5 & 9,0 & 48,02 \\
\hline
\end{tabular}


óleo bruto na prensagem. Para um nível de confiança de 95\% $(\mathrm{p} \leq 0,05)$ pode-se observar que os fatores significativos são temperatura e umidade.

Para o rendimento em óleo bruto pode-se observar que a interação entre temperatura e teor de umidade não influenciou significativamente na resposta $(\mathrm{p}>0,05)$. Retirando-se o único termo não significativo, foi feita uma Análise de Variância (ANOVA) com aplicação do teste F para a verificação da significância da regressão e geração dos modelos preditos, conforme resultados apresentados na Tabela 4 .

Como o $\mathrm{F}_{\text {calc }}$ é superior ao $\mathrm{F}_{\text {tab }}$ pode-se dizer que o modelo apresentou uma regressão significativa, com os respectivos graus de liberdade. Barros Neto, Scarminio e Bruns (2003) afirmam que uma regressão pode ser significativa do ponto de vista do teste $\mathrm{F}$, mas pode não servir para realizar previsões, uma vez que cobre uma faixa pequena da variação dos fatores estudados.

O critério estabelecido por Box e Wetz (1973), citados por Barros Neto, Scarminio e Bruns (2003), sugere que para uma regressão ser significativa não apenas estatisticamente, mas também para fins preditivos, o valor de $\mathrm{F}_{\text {calc }}$ para a regressão deve ser maior que o valor de $\mathrm{F}_{\mathrm{tab}}$.

A falta de ajuste do modelo não foi significativa, pois o $F_{\text {calc }}$ foi inferior ao $\mathrm{F}_{\mathrm{tab}}$, $\mathrm{o}$ que significa que não há evidência de falta de ajuste do modelo. $\mathrm{O}$ coeficiente de determinação $\left(\mathrm{R}^{2}\right)$ indica que a porcentagem da variação explicada pelo modelo foi de $91,3 \%$, enquanto somente $8,7 \%$ não foi explicada pelo modelo.

Pelas análises de regressão múltipla dos dados experimentais, obteve-se o modelo polinomial de primeira ordem, na forma não codificada (Equação 2). Não houve a necessidade de expansão do modelo para segunda ordem, com adição dos pontos axiais, já que a análise dos resultados indicou que o modelo é linear.

$\operatorname{Re} n d=36,70445+21,61103 * T+13,20674 * U$

O erro médio relativo apresentado pelo modelo foi de $20,8 \%$. A partir deste modelo construiu-se a superfície de resposta e as curvas em nível para o rendimento em óleo bruto da prensagem de grãos de amendoim (Figura 1).

Tabela 3. Efeito estimado, erro padrão e grau de significância estatística (p) para a extração de óleo de amendoim.

\begin{tabular}{lccc}
\hline \multicolumn{1}{c}{ Variáveis } & Efeito & Erro padrão & $\mathrm{p}$ \\
\hline Temperatura T (linear) & 43,2221 & 8,0155 & 0,0125 \\
Umidade U (linear) & 26,4135 & 8,0155 & 0,0460 \\
Interação T x U & 7,20368 & 8,0155 & 0,4350 \\
\hline
\end{tabular}

Tabela 4. Análise de variância (ANOVA) para a extração de óleo de amendoim.

\begin{tabular}{lcccccc}
\hline \multicolumn{1}{c}{$\begin{array}{c}\text { Fonte de } \\
\text { variação }\end{array}$} & $\begin{array}{c}\text { Soma } \\
\text { quadrática }\end{array}$ & $\begin{array}{c}\text { Graus de } \\
\text { liberdade }\end{array}$ & $\begin{array}{c}\text { Média } \\
\text { quadrática }\end{array}$ & $\mathrm{F}_{\text {calc }}$ & $\mathrm{F}_{\text {tab }}$ & $\mathrm{R}^{2}$ \\
\hline Regressão & $2,57 \mathrm{E}+03$ & 2 & $1,28 \mathrm{E}+03$ & 20,98 & 6,94 & 0,9130 \\
Resíduo & $2,45 \mathrm{E}+02$ & 4 & $6,12 \mathrm{E}+01$ & & & \\
Falta de ajuste & $5,63 \mathrm{E}+01$ & 2 & $2,81 \mathrm{E}+01$ & 0,30 & 19,00 & \\
Erro puro & $1,88 \mathrm{E}+02$ & 2 & $9,42 \mathrm{E}+01$ & & & \\
Total & $2,81 \mathrm{E}+03$ & 6 & & & & \\
\hline
\end{tabular}

Os maiores rendimentos em óleo bruto de amendoim, visualizados pela superfície de resposta, estão relacionados aos maiores valores de teor de umidade e de temperatura dos grãos durante a prensagem. Dentro da faixa de valores para temperatura e teor de umidade adotada neste estudo, pode-se afirmar que para temperaturas entre 50 e $65^{\circ} \mathrm{C}$ e teor de umidade entre 10 e 10,8\%, o rendimento em óleo será máximo.

$\mathrm{Na}$ literatura não foi encontrado nenhum estudo semelhante ao aqui desenvolvido. Mpagalile, Hanna e Weber (2006) avaliaram a prensagem de grãos de amendoim picados, a $12 \%$ de teor de umidade, sob um eixo aquecido a $60^{\circ} \mathrm{C}$, alcançando rendimento de $70 \%$ em óleo bruto, valor próximo ao máximo obtido neste trabalho.

A composição em ácidos graxos do óleo de amendoim está mostrada na Tabela 5.
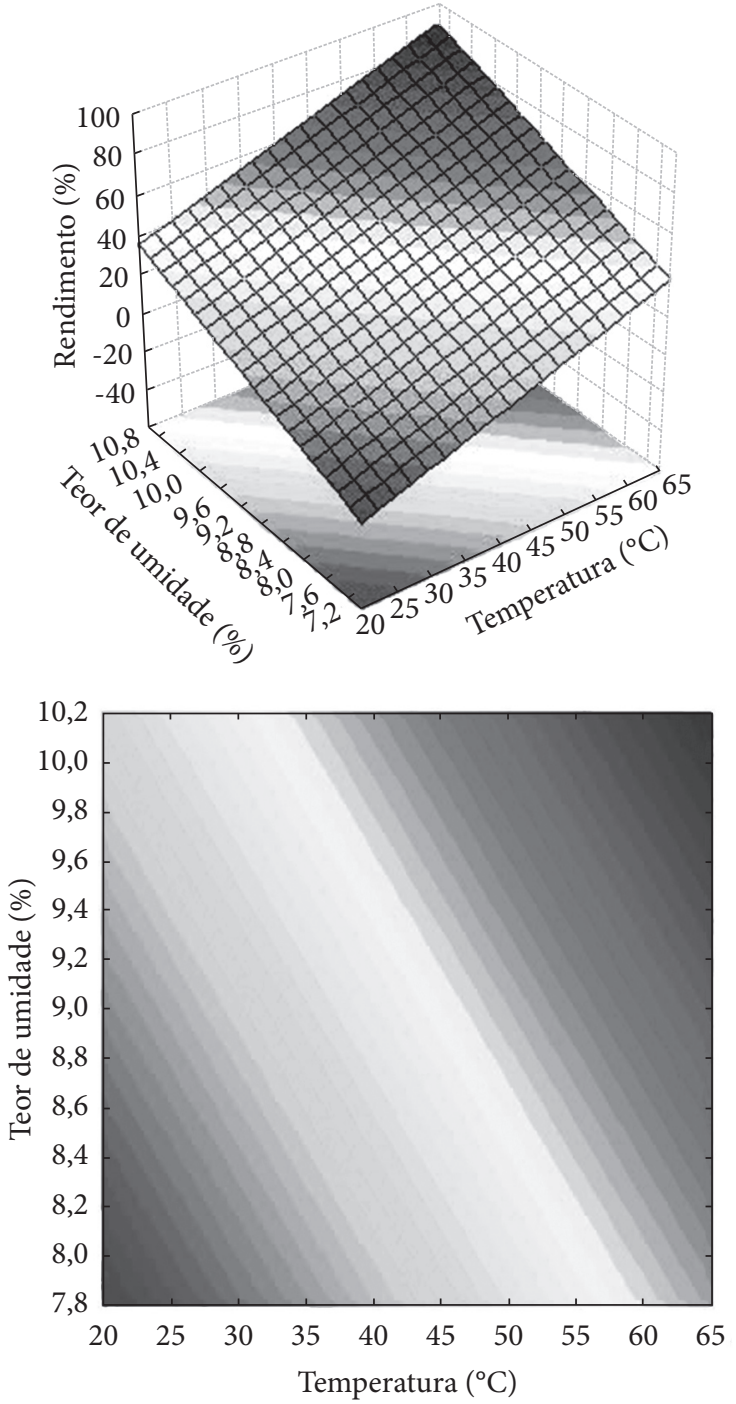

$80 \square 60 \square 40 \square 20 \square 0$

Figura 1. Superfície de resposta e curvas em nível para o rendimento de óleo bruto em função do teor de umidade e temperatura de grãos de amendoim extraídos por prensagem mecânica contínua. 
Tabela 5. Composição em ácidos graxos do óleo bruto de amendoim.

\begin{tabular}{|c|c|c|}
\hline Ácidos graxos & $\begin{array}{l}\text { Massa molar } \\
\left(\text { g.mol }{ }^{-1}\right)\end{array}$ & $\begin{array}{c}\text { Concentraçãa } \\
(\%)\end{array}$ \\
\hline C16:0 Palmítico & 226,445 & 10,2 \\
\hline C17:0 Margárico & 240,471 & 0,1 \\
\hline C18:0 Esteárico & 254,498 & 2,0 \\
\hline 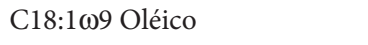 & 252,482 & 46,7 \\
\hline 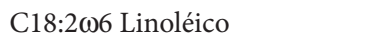 & 250,467 & 32,0 \\
\hline C20:0 Araquídico & 282,552 & 1,2 \\
\hline 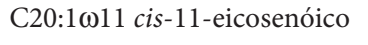 & 280,536 & 1,4 \\
\hline C22:0 Behênico & 310,605 & 3,7 \\
\hline C22:1 Erúcico & 308,590 & 0,1 \\
\hline C24:0 Lignocérico & 338,659 & 2,2 \\
\hline $\begin{array}{l}\text { C22:5 } 133 \text { cis }-7,10,13,16,19 \\
\text { - docosapentaenóico (DPA) }\end{array}$ & 300,526 & 0,4 \\
\hline
\end{tabular}

Fernandez e Rosolem (1998) encontraram a seguinte composição em ácidos graxos para o óleo de amendoim: 10,52\% de palmítico, 2,90\% de esteárico, 41,28\% de oléico, 37,22\% de linoléico, 1,61\% de araquídico, 3,48\% de behênico e 1,48\% de lignocérico. A composição encontrada para o óleo bruto de amendoim está próxima da literatura, indicando que o processamento adotado não alterou suas características quanto aos ácidos graxos. Com base nos valores da Tabela 5, a massa molar do óleo bruto de amendoim obtido foi de 800,77 g.mol ${ }^{-1}$. Esta informação foi importante para o cálculo do rendimento em biodiesel (PIGHINELLI, 2007).

$\mathrm{Na}$ Tabela 6 estão os resultados das demais análises de caracterização feitas no óleo.

Para o óleo de amendoim refinado, a faixa de variação do índice de saponificação é de 188 a 195 mgKOH.g-1 e a densidade relativa entre 0,910 e 0,915 (WEISS, 1983; FREIRE et al., 2005). O índice de peróxidos encontrado para o óleo bruto apresentou um valor bem inferior ao citado na literatura. Chu e Kung (1998) encontraram um índice de peróxidos de 7,70 meq. $\mathrm{kg}^{-1}$, enquanto Chu e Hsu (1999) encontraram um valor de 3,28 meq. $\mathrm{kg}^{-1}$. Quanto ao parâmetro cor, Lovibond e Weiss (1983) citam uma variação entre 16 e 25 no amarelo e entre 1 e 2 no vermelho.

A torta obtida para cada ponto experimental foi pesada e caracterizada quanto ao seu teor de lipídios (Tabela 7). Os altos teores de óleo indicam a necessidade de uma segunda prensagem ou um tratamento com solventes, para contribuir com um maior rendimento do processamento.

\section{Conclusões}

Para a prensagem dos grãos de amendoim, tanto a temperatura quanto o teor de umidade afetaram de forma positiva o rendimento em óleo bruto.

Temperaturas entre 50 e $65^{\circ} \mathrm{C}$ e teor de umidade entre $10 \mathrm{e}$ $10,8 \%$ corresponderam a rendimentos máximos.

A caracterização do óleo bruto de amendoim é próxima a do óleo refinado, indicando que a prensagem a frio não afetou a qualidade do óleo.
Tabela 6. Caracterização do óleo bruto de amendoim.

\begin{tabular}{|c|c|}
\hline Análises & Resultados \\
\hline Índice de iodo - Método de Wijs (cgl.g-1) & 94,85 \\
\hline Índice de refração $\left(20^{\circ}\right)$ & 1,46 \\
\hline Índice de saponificação (mg KOH-g-1) & 184,54 \\
\hline Matéria insaponificável $\left(\mathrm{g} .100 \mathrm{~g}^{-1}\right)$ & 0,29 \\
\hline Densidade relativa $\left(20^{\circ} \mathrm{C}\right)$ & 0,92 \\
\hline Ácidos graxos livres (g.100 g ${ }^{-1}$ de acido oléico) & 0,32 \\
\hline Índice de peróxidos (meq. $\mathrm{kg}^{-1}$ ) & 1,20 \\
\hline Teor de umidade (g.100 $\mathrm{g}^{-1}$ ) & 0,06 \\
\hline Cor Lovibond (cubeta 5 1/4”) & $\begin{array}{c}2 \text { amarelo } \\
0,1 \text { vermelho }\end{array}$ \\
\hline
\end{tabular}

Tabela 7. Rendimento e teor de lipídios da torta resultante da prensagem.

\begin{tabular}{ccc}
\hline Ensaios & Torta (\%) & Lipídios na torta (\%) \\
\hline 1 & 95,7 & 43,82 \\
2 & 81,7 & 36,15 \\
3 & 88,2 & 37,27 \\
4 & 64,5 & 21,60 \\
5 & 89,2 & 43,71 \\
6 & 82,8 & 37,06 \\
7 & 75,3 & 31,29 \\
\hline
\end{tabular}

\section{Agradecimentos}

À UNICAMP - Universidade Estadual de Campinas; à FAPESP - Fundação de Amparo à Pesquisa do Estado de São Paulo; ao CNPq - Conselho Nacional de Desenvolvimento Científico e Tecnológico; e ao ITAL - Instituto de Tecnologia de Alimentos.

\section{Referências bibliográficas}

AOCS - American Oil Chemists Society. Official methods and recommended practices of the AOCS. Champaign: A.O.C.S., 1998.

Official methods of analysis of A.O.A.C. international. 16 ed. Arlington: A.O.A.C., 1998.

BARROS NETO, B.; SCARMÍNIO, I. S.; BRUNS, R. E. Planejamento e Otimização de Experimentos. Campinas: Editora da UNICAMP, 2003. 299 p.

BOX, G. E. P.; WETZ, J. Criteria for judging adequacy of estimation by an approximate response function. Wisconsin: University of Wisconsin, 1973. Tec. Report. n. 9.

BOX, G. E. P.; HUNTER, W. G.; HUNTER, J. S. Statistics for experimenters. An introduction designs, data analysis and model building. New York: Wiley, 1978.

CHU. Y.; HSU, H. Effects of antioxidants on peanut oil stability. Food Chemistry, v. 66, n. 1, p. 29-34, 1999.

CHU, Y.; KUNG, Y. A study on vegetable oil blends. Food Chemistry, v. 62, n. 2, p. 191-195, 1998.

FERNANDEZ, E. M.; ROSOLEM, C. A. Ácidos graxos e proteína em grãos de amendoim em função da calagem e do método de secagem. Bragantia, v. 57, n. 1, 1998 . Disponível em: $<$ http://www.scielo.br/scielo.php?script=sci_arttext\&pid=S0006$87051998000100004 \& \operatorname{lng}=\mathrm{en} \& \mathrm{nrm}=\mathrm{iso}>$. Acesso em: $30 \mathrm{jul}$. 2005. 
FREITAS, S. M. et al. Evolução do Mercado brasileiro de amendoim. In: SANTOS, R. C. (Ed). O agronegócio do amendoim no Brasil. Campina Grande: Embrapa Algodão, 2005. p. 17-44.

GODOY, I. J. et al. Cultivares de amendoim IAC: novas opções para o mercado de confeitaria. Campinas: Instituto Agronômico, 2003. 10 p. (Boletim Técnico).

MPAGALILE, J. J.; HANNA, M. A.; WEBER, R. Design and testing of a solar photovoltaic operated multi-seeds oil press. Renewable Energy, v. 31, n. 12, p. 1855-1866, 2006.

MYERS, R. H.; MONTGOMERY, D. C. Response Surface Methodology. New York: John Wiley \& Sons Inc., 2002. p. 1-17.

OHLSON, I. S. R. Modern processing of rapeseed. Journal of the American Oil Chemists' Society, v. 69, p. 195-198, 1992.

PIGHINELLI, A. L. M. T. Extração mecânica de óleos de amendoim e de girassol para a produção de biodiesel via catálise básica. Campinas, 2007. 93 f. Dissertação - (Mestrado em Engenharia Agrícola), Faculdade de Engenharia Agrícola, Universidade Estadual de Campinas.

SANTOS, R. C.; GODOY, J. I.; FÁVERO, A. P. Melhoramento do amendoim. In: SANTOS, R. C. (Ed). O agronegócio do amendoim no Brasil. Campina Grande: Embrapa Algodão, 2005. p. 125-172.
SINGH, M. S. et al. Development of mathematical models to predict sunflower oil expression. Transactions of the ASAE, p. 1190-1194, 1984.

SINGH, J.; BARGALE, P. C. Mechanical expression of oil from linseed (Linum usitatissimum L.). Journal Oilseeds Research, v. 7, n. 2, p. 106-110, 1990.

SINGH, J.; BARGALE, P. C. Development of a small capacity double stage compression screw press for oil expression. Journal of Food Engineering, v. 43, p. 75-82, 2000.

SINGH, K. K. et al. Influence of moisture content and cooking on screw pressing of crambe seed. Journal of the American Oil Chemists Society, v. 79, p. 165-170, 2002.

SRIKANTHA, P. V. R. A search for an appropriate technology for village oil industry. India: AINDA; Lucknow (India) Publications, 1980.

STATISTICA. Data analysis software system. Versão 6.0. Tulsa: StatSoft, Inc., 2001.

WEISS, E. A. Oilseed processing and products. In: Oilseed crops. 1 ed. London: Longman, 1983. p. 528-596. 